Géometrie différentielle / Differential Geometry

\title{
EIGENVALUE ESTIMATES FOR THE DIRAC OPERATOR AND HARMONIC 1-FORMS OF CONSTANT LENGTH
}

\author{
ANDREI MOROIANU AND LIVIU ORNEA
}

\begin{abstract}
.
We prove that on a compact $n$-dimensional spin manifold admitting a non-trivial harmonic 1 -form of constant length, every eigenvalue $\lambda$ of the Dirac operator satisfies the inequality $\lambda^{2} \geq \frac{n-1}{4(n-2)} \inf _{M}$ Scal. In the limiting case the universal cover of the manifold is isometric to $\mathbb{R} \times N$ where $N$ is a manifold admitting Killing spinors.
\end{abstract}

\section{Estimations de valeurs propres pour l'opérateur de Dirac et 1-formes harmoniques de longueur constante}

RÉSUMÉ.

Nous démontrons que toute valeur propre $\lambda$ de l'opérateur de Dirac d'une variété spinorielle compacte, de dimension $n$, qui admet une 1-forme harmonique non-triviale de longueur constante vérifie l'inégalité $\lambda^{2} \geq \frac{n-1}{4(n-2)} \inf _{M}$ Scal. Dans le cas limite le revêtement universel de la variété est isométrique à $\mathbb{R} \times N$ où $N$ est une variété admettant des spineurs de Killing.

\section{INTRODUCTION}

Let $\left(M^{n}, g\right)$ be a compact spin manifold of (real) dimension $n$. For positive scalar curvature Scal $:=\operatorname{Scal}(M, g)$, every eigenvalue $\lambda$ of the Dirac operator $D$ satisfy the well-known Friedrich inequality [4]:

$$
\lambda^{2} \geq \frac{n}{4(n-1)} \inf _{M} \mathrm{Scal} \text {. }
$$

There exist manifolds on which the inequality is indeed sharp: the limiting case is equivalent to the existence of a Killing spinor, i.e. a spinor $\Psi$ satisfying the equation

$$
\nabla_{X} \Psi+\frac{\lambda}{n} X \cdot \Psi=0
$$

Key words and phrases. Spin manifold, eigenvalues of Dirac operator, harmonic form, parallel form, Killing spinor.

2000 Mathematics Subject Classification. 53C27, 58B40.

The authors are members of EDGE, Research Training Network HRPN-CT-2000-00101, supported by the European Human Potential Programme. 
On the other hand, Hijazi noticed that a manifold admitting a parallel $k$-form, $k \neq 0, n$, carries no Killing spinors [5]. Consequently, Friedrich's inequality cannot be sharp on manifolds with geometric structures which support parallel forms. Various authors have obtained sharp improvements of (1) on Kähler and quaternionic Kähler manifolds (see [6], [7], [8]).

Another recent improvement of Friedrich inequality in this latter direction was found by Alexandrov, Grantcharov and Ivanov. They proved in [1] that the existence of a parallel 1-form on $M^{n}, n \geq 3$, implies the inequality

$$
\lambda^{2} \geq \frac{n-1}{4(n-2)} \inf _{M} \text { Scal } .
$$

The universal covering space of the manifolds appearing in the limiting case was also described.

In this note we generalize the above result showing that (3) can be derived only from the existence of a harmonic 1-form with constant length:

Theorem 1. Inequality (3) holds on any compact spin manifold $\left(M^{n}, g\right), n \geq 3$, admitting a non-trivial harmonic 1-form $\theta$ of constant length. The limiting case is obtained if and only if $\theta$ is parallel and the eigenspinor $\Psi$ corresponding to the smallest eigenvalue of the Dirac operator satisfies the following Killing type equation:

$$
\nabla_{X} \Psi+\frac{\lambda}{n-1}(X \cdot \Psi-\langle X, \theta\rangle \theta \cdot \Psi)=0
$$

after rescaling $\theta$ to unit length.

The condition for the norm of the 1-form to be constant is essential, in the sense that the topological constraint alone - the existence of a non-trivial harmonic 1-form - does not allow any improvement of Friedrich's inequality.

Indeed, motivated by a conjecture appearing in an earlier version of this note, Bär and Dahl [3] have constructed, on any compact spin manifold $M^{n}$ and for every positive real number $\epsilon$, a metric $g_{\epsilon}$ on $M$ with the property that $\operatorname{Scal}_{g_{\epsilon}} \geq n(n-1)$ and such that the first eigenvalue of the Dirac operator satisfies $\lambda_{1}^{2}\left(D_{\epsilon}\right) \leq \frac{n^{2}}{4}+\epsilon$. This construction clearly shows that no improvement of Friedrich's inequality can be obtained under purely topological restrictions.

Acknowledgment. L.O. thanks the Centre of Mathematics of the Ecole Polytechnique (Palaiseau) for hospitality during March-May 2003 when this research was initiated.

\section{The MAIN INEQUALITY}

Let $\theta$ be a 1 -form of unit length on a spin manifold $\left(M^{n}, g\right)$ and let $\Psi$ be an arbitrary spinor field on $M$. We identify 1 -forms with vector fields by means of the scalar product that we denote with $\langle$,$\rangle .$ 
Consider the following "twistor-like" operator $T: T M \otimes \Sigma M \rightarrow \Sigma M$

$$
T_{X} \Psi=\nabla_{X} \Psi+\frac{1}{n-1} X \cdot D \Psi-\frac{1}{n-1}\langle X, \theta\rangle \theta \cdot D \Psi-\langle X, \theta\rangle \nabla_{\theta} \Psi
$$

where the dot $\cdot$ denotes Clifford multiplication. A simple calculation yields

$$
|T \Psi|^{2}=|\nabla \Psi|^{2}-\frac{1}{n-1}|D \Psi|^{2}-\left|\nabla_{\theta} \Psi\right|^{2}+\frac{2}{n-1}\left\langle D \Psi, \theta \cdot \nabla_{\theta} \Psi\right\rangle .
$$

From now on we will suppose that $\theta$ is harmonic, $M$ is compact with volume element $d \mu$ and has positive scalar curvature Scal, and $\Psi$ is an eigenspinor of the Dirac operator $D$ of $M$ corresponding to the least eigenvalue (in absolute value), say $\lambda$. We let $\left\{e_{i}\right\}, i=1, \ldots, n$ denote a local orthonormal frame on $M$.

The harmonicity of $\theta$ implies the following useful relation:

$$
D(\theta \cdot \Psi)=-\theta \cdot D \Psi-2 \nabla_{\theta} \Psi .
$$

Indeed, one may write:

$$
\begin{aligned}
D(\theta \cdot \Psi) & =\sum e_{i} \cdot \nabla_{e_{i}}(\theta \cdot \Psi)=\sum e_{i} \cdot\left(\nabla_{e_{i}} \theta\right) \cdot \Psi+e_{i} \cdot \theta \cdot \nabla_{e_{i}} \Psi \\
& =(d \theta+\delta \theta) \cdot \Psi+e_{i} \cdot \theta \cdot \nabla_{e_{i}} \Psi \\
& =-\theta \cdot \sum e_{i} \cdot \nabla_{e_{i}} \Psi-2\left\langle e_{i}, \theta\right\rangle \nabla_{e_{i}} \Psi \\
& =-\theta \cdot D \Psi-2 \nabla_{\theta} \Psi .
\end{aligned}
$$

Taking the square norm in (6) yields

$$
|D(\theta \cdot \Psi)|^{2}=|\theta \cdot D \Psi|^{2}+4\left|\nabla_{\theta} \Psi\right|^{2}-4\left\langle D \Psi, \theta \cdot \nabla_{\theta} \Psi\right\rangle,
$$

By integration over $M$ in (5), using (7) to express the last term in the right hand side of (5), and the Lichnerowicz formula $D^{2}=\nabla^{*} \nabla+\frac{1}{4}$ Scal, we get

$$
\begin{aligned}
\int_{M}|T \Psi|^{2} d \mu & =\int_{M}\left\{\frac{n-2}{n-1}|D \Psi|^{2}-\frac{1}{4} \operatorname{Scal}|\Psi|^{2}-\frac{n-3}{n-1}\left|\nabla_{\theta} \Psi\right|^{2}\right. \\
& \left.-\frac{1}{2(n-1)}\left(|D(\theta \cdot \Psi)|^{2}-|\theta \cdot D \Psi|^{2}\right)\right\} d \mu .
\end{aligned}
$$

The term in the last bracket of the integrand is clearly positive since, from the choice of $\lambda$ to be minimal, we have from the classical Rayleigh inequality

for every $\Phi$. In particular, for $\Phi=\theta \cdot \Psi$ this reads

$$
\lambda^{2} \leq \frac{\int_{M}|D \Phi|^{2} d \mu}{\int_{M}|\Phi|^{2} d \mu}
$$

$$
\int_{M}|D(\theta \cdot \Psi)|^{2} d \mu \geq \lambda^{2} \int_{M}|\theta \cdot \Psi|^{2} d \mu=\lambda^{2} \int_{M}|\Psi|^{2} d \mu=\int_{M}|D \Psi|^{2} d \mu=\int_{M}|\theta \cdot D \Psi|^{2} d \mu .
$$

Thus (8) gives

$\int_{M}\left(\frac{n-2}{n-1} \lambda^{2}-\frac{1}{4} \mathrm{Scal}\right)|\Psi|^{2} d \mu=\int_{M}|T \Psi|^{2}+\frac{n-3}{n-1}\left|\nabla_{\theta} \Psi\right|^{2}+\frac{1}{2(n-1)}\left(|D(\theta \cdot \Psi)|^{2}-|\theta \cdot D \Psi|^{2}\right) d \mu \geq 0$,

which immediately implies the first statement of Theorem 1. 


\section{The Limiting CASE}

Suppose now that equality is reached in (3) for the eigenvalue $\lambda$ with corresponding eigenspinor $\Psi$. Then $T \Psi=0$. Contracting with $e_{i}$ :

$$
\sum e_{i} \cdot \nabla_{e_{i}} \Psi+\frac{\lambda}{n-1} \sum e_{i} \cdot e_{i} \cdot \Psi-\frac{\lambda}{n-1} \sum e_{i} \cdot\left\langle e_{i}, \theta\right\rangle \theta \cdot \Psi-e_{i} \cdot\left\langle e_{i}, \theta\right\rangle \nabla_{\theta} \Psi=0,
$$

gives $\theta \cdot \nabla_{\theta} \Psi=0$, so $\nabla_{\theta} \Psi=0$ (for $n>3$ this follows directly from the vanishing of the integral $\left.\int_{M} \frac{n-3}{n-1}\left|\nabla_{\theta} \Psi\right|^{2} d \mu\right)$. Thus $\Psi$ satisfies the Killing type equation

$$
\nabla_{X} \Psi=a X \cdot \Psi-a\langle X, \theta\rangle \theta \cdot \Psi, \quad a=-\frac{\lambda}{n-1} .
$$

In order to show that $\theta$ is parallel, we first compute the spin curvature operator $\mathcal{R}_{Y, X}=$ $\left[\nabla_{Y}, \nabla_{X}\right]-\nabla_{[Y, X]}$ acting on $\Psi$. We have successively :

$$
\begin{aligned}
\frac{1}{a} \nabla_{Y} \nabla_{X} \Psi & =\nabla_{Y} X \cdot \Psi+a X \cdot(Y-\langle Y, \theta\rangle \theta) \cdot \Psi-\left\langle\nabla_{Y} X, \theta\right\rangle \theta \cdot \Psi \\
& -\langle X, \theta\rangle \nabla_{Y} \theta \cdot \Psi-\left\langle X, \nabla_{Y} \theta\right\rangle \theta \cdot \Psi-a\langle X, \theta\rangle \theta \cdot(Y-\langle Y, \theta\rangle \theta) \cdot \Psi \\
\frac{1}{a} \mathcal{R}_{Y, X} \cdot \Psi & =a(X \cdot Y-Y \cdot X) \cdot \Psi-a(\langle Y, \theta\rangle X \cdot \theta-\langle X, \theta\rangle Y \cdot \theta) \cdot \Psi \\
& +\left(\left\langle Y, \nabla_{X} \theta\right\rangle-\left\langle X, \nabla_{Y} \theta\right\rangle\right) \theta \cdot \Psi+\left(\langle Y, \theta\rangle \nabla_{X} \theta-\langle X, \theta\rangle \nabla_{Y} \theta\right) \cdot \Psi \\
& +a(\langle Y, \theta\rangle \theta \cdot X-\langle X, \theta\rangle \theta \cdot Y) \cdot \Psi \\
& =a(X \cdot Y-Y \cdot X) \cdot \Psi+2 a(\langle Y, \theta\rangle \theta \cdot X-\langle X, \theta\rangle \theta \cdot Y) \cdot \Psi \\
& +\left(\left\langle Y, \nabla_{X} \theta\right\rangle-\left\langle X, \nabla_{Y}\right\rangle\right) \theta \cdot \Psi+\left(\langle Y, \theta\rangle \nabla_{X} \theta-\langle X, \theta\rangle \nabla_{Y} \theta\right) \cdot \Psi
\end{aligned}
$$

Using again the harmonicity of $\theta$ we easily derive :

$$
\begin{aligned}
\frac{1}{2 a} \operatorname{Ric}(X) \cdot \Psi & =\frac{1}{a} \sum e_{i} \cdot \mathcal{R}_{e_{i}, X} \Psi= \\
& =2(n-2) a(X-\langle X, \theta\rangle \theta) \cdot \Psi+2 \theta \cdot \nabla_{X} \theta \cdot \Psi-2\left\langle X, \nabla_{\theta} \theta\right\rangle \Psi
\end{aligned}
$$

But $\nabla_{\theta} \theta=0$ because $\theta$ is unitary and closed. Indeed, for any vector field $Y$ :

$$
\left\langle Y, \nabla_{\theta} \theta\right\rangle=d \theta(\theta, Y)-\langle\theta, Y\rangle=0 .
$$

Hence, taking $X=\theta$ in (10), we obtain $\operatorname{Ric}(\theta)=0$. Then, as $\theta$ is harmonic, the Bochner formula assures that $\theta$ is parallel. This completes the proof of Theorem 1.

Since the 1 -form $\theta$ has to be parallel in the limiting case, we can apply Theorem 3.1 in [1] to determine the universal cover of $M$. In fact, as proved in loc. cit., this is isometric to a Riemannian product $\mathbb{R} \times N$, where $N$ is a spin manifold carrying a real Killing spinor, hence can be described by Bär's classification [2]. Finally, $M$ turns out to be a suspension of an isometry of $N / \Gamma$ (a finite quotient of $N$ ) over the circle. 


\section{REFERENCES}

[1] B. Alexandrov, G. Grantcharov, S. Ivanov, An estimate for the first eigenvalue of the Dirac operator on compact Riemannian spin manifold admitting parallel one-form, J. Geom. Phys. 28 (1998), 263-270.

[2] C. Bär, Real Killing spinors and holonomy, Comm. Math. Phys. 154 (1993), 509-521.

[3] C. Bär, M. Dahl, The first Dirac eigenvalue on manifolds with positive scalar curvature, Proc. Amer. Math. Soc. 132 (2004), 3337-3344.

[4] T. Friedrich, Der erste Eigenwert des Dirac Operators eines kompakten Riemmanschen Mannifaltigkeit nichtnegativer Skalarkrümung, Math. Nachr. 97 (1980), 117-146.

[5] O. Hijazi, Opérateurs de Dirac sur les variétés Riemanniennes. Minoration des valeurs propres, Thèse de 3-ème cycle, Ecole Polytechnique, 1984.

[6] K.-D. Kirchberg, An estimation for the first eigenvalue of the Dirac operator on closed Kähler manifolds of positive scalar curvature, Ann. Global Anal. Geom. 3 (1986), 291-325.

[7] K.-D. Kirchberg, The first Eigenvalue of the Dirac Operator on Kähler Manifolds, J. Geom. Phys. 7 (1990) 449-468.

[8] W. Kramer, U. Semmelmann, G. Weingart Eigenvalue estimates for the Dirac operator on quaternionic Kähler manifolds, Math. Z. 230 (1999), 727-751.

Centre de Mathémathiques, Ecole Polytechnique, 91128 Palaiseau Cedex, France

E-mail address: am@math.polytechnique.fr

University of Bucharest, Faculty of Mathematics, 14 Academiei str., 70109 Bucharest, Romania

E-mail address: Liviu.Ornea@imar.ro 